\title{
Spin quantum well-like behavior in single-crystal $\mathrm{Gd}_{0.75} \mathrm{La}_{0.25} \mathrm{FeO}_{3}$
}

\author{
Xin Wen ${ }^{1}$, Zhigang Song ${ }^{1}$, Iftikhar Ahmed Malik ${ }^{2}$, Yifei Fang ${ }^{3}$, Wenyun Yang ${ }^{1,4}$, Jingzhi Han ${ }^{1,4}$, \\ Shunquan Liu ${ }^{1,4}$, Honglin $\mathrm{Du}^{1,4}$, Shixun $\mathrm{Cao}^{5}$, Jinxing Zhang ${ }^{2}$, Xiangqun Zhang ${ }^{6}$, Zhaohua Cheng ${ }^{6}$, \\ Yanglong Hou ${ }^{4,7^{*}}$ and Jinbo Yang ${ }^{1,5^{*}}$
}

Rare earth orthoferrites $\left(\mathrm{RFeO}_{3}, \mathrm{R}=\mathrm{Y}, \mathrm{Gd}, \mathrm{Sm}, \mathrm{Nd}\right)$ have recently attracted much attention due to their novel magnetic and electrical properties such as magnetoelectrics, magneto-dielectrics, and magneto-optics [1-3]. The complex interactions between the two sublattices of $\mathrm{R}^{3+}$ and $\mathrm{Fe}^{3+}$ in $\mathrm{RFeO}_{3}$ provide the feasibility of controlling spins in these solids, which makes them promising candidates for the spintronics [4]. A major challenge is to develop a kind of material in which favorable properties such as stability, tunability, and non-volatility [5] can be combined. In this paper, we prepared a high-quality single-crystal of $\mathrm{Gd}_{0.75} \mathrm{La}_{0.25} \mathrm{FeO}_{3}$ and investigated its magnetic properties from $2 \mathrm{~K}$ to room temperature. Different from other $\mathrm{RFeO}_{3}$ orthoferrites $(\mathrm{R}=$ rare earth, such as Gd [6], La [7], Er [8], Sm [9,10], and Tm [11]), the weak-ferromagnetic (wFM) moments of the singlecrystal of $\mathrm{Gd}_{0.75} \mathrm{La}_{0.25} \mathrm{FeO}_{3}$ are discrete and have only two states: the same in magnitude but in opposite direction along the ferromagnetic axis, thus being not able to rotate randomly. The AFM coupling between Gd and Fe sublattices is broken in $\mathrm{Gd}_{0.75} \mathrm{La}_{0.25} \mathrm{FeO}_{3}$, which leads to a spin-flip. The moment of the Gd sublattice behaves in the same direction with the moment of Fe sublattice until the external magnetic field is large enough. On the other hand, different from common materials whose coercivity often varies with the temperature [12-18], the coercivity in $\mathrm{Gd}_{0.75} \mathrm{La}_{0.25} \mathrm{FeO}_{3}$ is nearly constant from 50 to $350 \mathrm{~K}$, avoiding being influenced by temperature in the application. These features give $\mathrm{Gd}_{0.75} \mathrm{La}_{0.25} \mathrm{FeO}_{3}$ great potential as a convenient and reliable material for information storage.

The single-phase sample of $\mathrm{Gd}_{0.75} \mathrm{La}_{0.25} \mathrm{FeO}_{3}$ was prepared by a solid-state method in a floating zone furnace (see Supplementary information). Fig. 1a illustrates the crystallographic structure of $\mathrm{Gd}_{0.75} \mathrm{La}_{0.25} \mathrm{FeO}_{3}$ and the magnetic structure of $\mathrm{Fe}$ atoms above the antiferromagnetic (AFM) ordering temperature of Gd. The $\mathrm{X}$-ray intensity profiles of the powdered single crystal (Fig. 1b) verify the successful preparation of $\mathrm{Gd}_{0.75} \mathrm{La}_{0.25^{-}}$ $\mathrm{FeO}_{3}$ with high crystalline quality (Fig. S1). All the peaks in the diffraction pattern can be indexed well with the typical perovskite structure having a monoclinic group of Pnma [19]. No visible structure phase transition is observed in the temperature range of 5-300 K (Fig. S2).

In our work, all the magnetic measurements were performed along the $b$-axis of the crystal (Fig. S1). $\mathrm{Gd}_{0.75} \mathrm{La}_{0.25} \mathrm{FeO}_{3}$ was first cooled to $2 \mathrm{~K}$ under a magnetic field of 0 Oe in the zero-field-cooled (ZFC) measurement and 300 Oe in the field-cooled (FC) measurement. The magnetization curves were then obtained under a measuring magnetic field of $50 \mathrm{Oe}$. The similar shapes and data values of ZFC and FC curves (Fig. 2a) indicate that $\mathrm{Gd}_{0.75} \mathrm{La}_{0.25} \mathrm{FeO}_{3}$ is not sensitive to the initial cooling process, different from other reported $\mathrm{ABO}_{3}$ orthoferrites $[6,10,20]$. The spin ordering temperature (Néel temperature $\left(T_{\mathrm{N}}\right)$ ) of the $\mathrm{Gd}$ sublattice is $3 \mathrm{~K}$, while the $\mathrm{Fe}$ sublattice shows an AFM ordering $\left(T_{\mathrm{N}}^{\mathrm{Fe}}=695 \mathrm{~K}\right)$ with a wFM of $0.06 \mu_{\mathrm{B}}$ per formula unit (f.u.) (Fig. 2b), likely due

\footnotetext{
${ }^{1}$ State Key Laboratory for Mesoscopic Physics and School of Physics, Peking University, Beijing 100871, China

2 Department of Physics, Beijing Normal University, Beijing 100875, China

${ }^{3}$ Department of Physics, Fudan University, Shanghai 200433, China

${ }^{4}$ Beijing Key Laboratory for Magnetoelectric Materials and Devices, Beijing 100871, China

${ }^{5}$ Department of Physics, Shanghai University, Shanghai 200444, China

${ }^{6}$ State Key Laboratory of Magnetism, Institute of Physics, Chinese Academy of Sciences, Beijing 100080, China

${ }^{7}$ Department of Materials Science and Engineering, College of Engineering, Peking University, Beijing 100871, China

* Corresponding authors (emails: hou@pku.edu.cn (Hou Y); jbyang@pku.edu.cn (Yang J))
} 

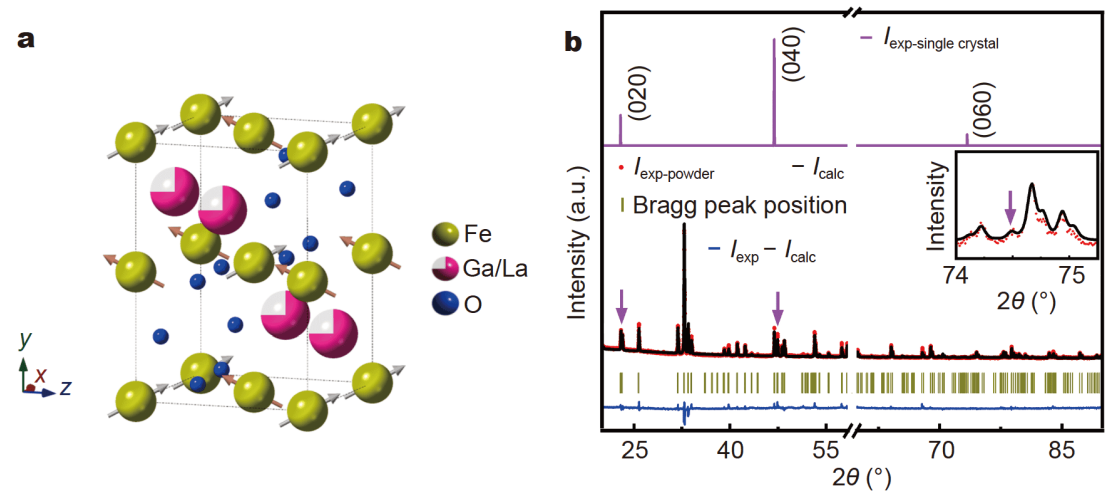

Figure 1 (a) Orthorhombic atomic structure and the magnetic structure of Fe atoms in $\mathrm{Gd}_{0.75} \mathrm{La}_{0.25} \mathrm{FeO}_{3}$. The arrows represent the directions of magnetic moments. (b) X-ray diffraction (XRD) patterns of the single-crystal $\mathrm{Gd}_{0.75} \mathrm{La}_{0.25} \mathrm{FeO}_{3}$ and powdered single-crystal at room temperature.
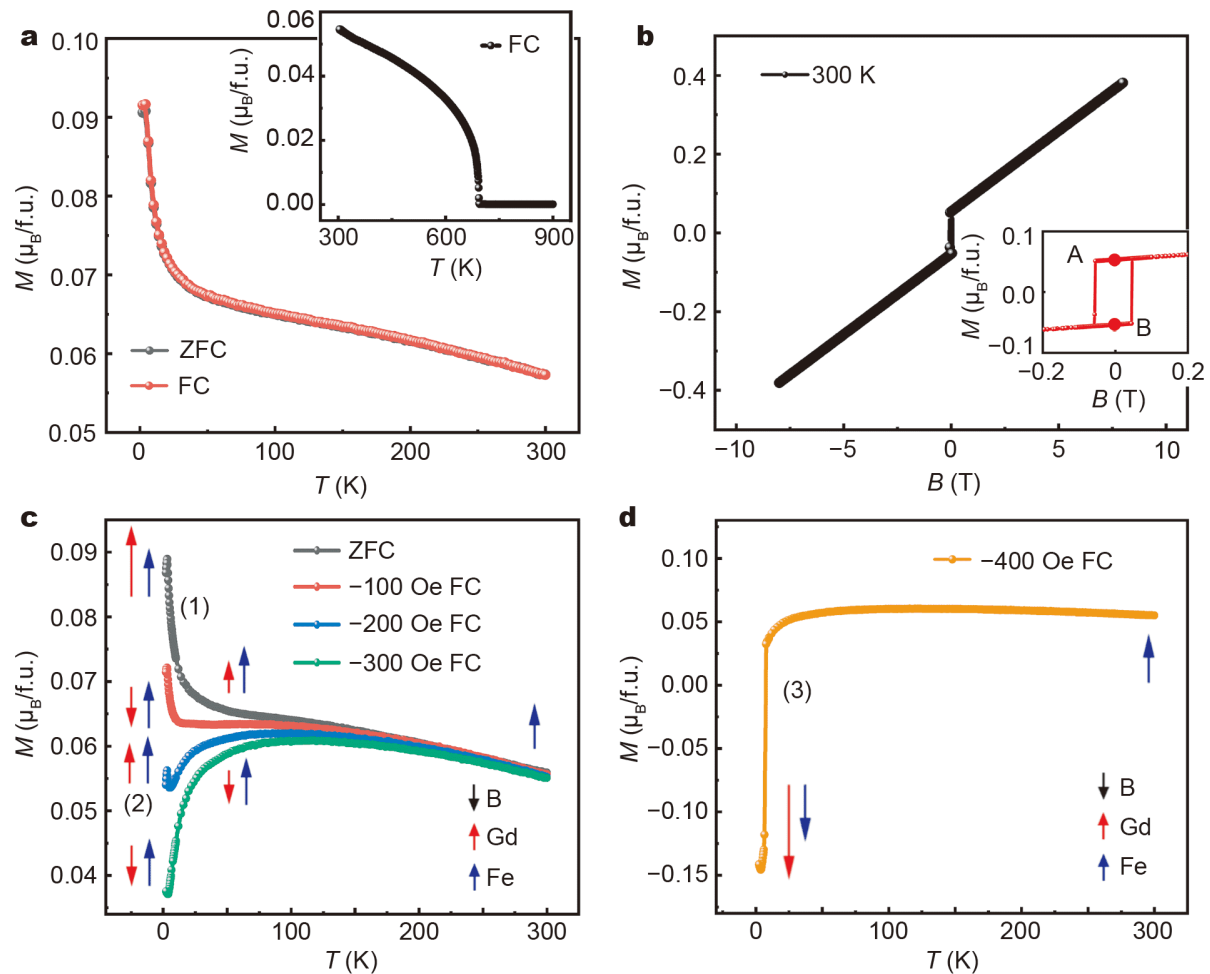

Figure 2 (a) Temperature dependence of magnetization after $\mathrm{ZFC}$ and $\mathrm{FC}$ in $\mathrm{Gd}_{0.75} \mathrm{La}_{0.25} \mathrm{FeO}_{3}$ after demagnetization. (b) The hysteresis loop at $300 \mathrm{~K}$. The inset shows the magnified view of the hysteresis loop where points $\mathrm{A}$ and $\mathrm{B}$ represent the original status when the external magnetic field is zero. (c, d) Temperature dependence of magnetization measured under different applied magnetic fields of (c) $0,-100,-200,-300$ Oe and (d) -400 Oe after the positive magnetization.

to the Dzyaloshinskii-Moriya (DM) interaction [21].

To investigate the evolution of magnetization, we kept the direction of the applied magnetic field $B$ downward and referred the magnitudes of fields to the absolute value (Fig. $2 \mathrm{c}$ and d). The corresponding directions of applied magnetic field $B, \mathrm{Fe}$ moments and $\mathrm{Gd}$ moments have been illustrated with arrows in the diagram of Fig. $2 \mathrm{c}$ and d. The $\mathrm{Gd}_{0.75} \mathrm{La}_{0.25} \mathrm{FeO}_{3}$ crystal was then magnetized un- der a large magnetic field so that the magnetization was fixed at the magnetic state of A or B shown in Fig. 2b. We also obtained $M-T$ curves in the FC cooling (FCC) processes when the magnetic fields were applied either antiparallel to the $b$-axis in Fig. $2 c$ or parallel to the $b$-axis in Fig. S3. The negative and positive signs herein represent the magnetic moments in the downward and upward directions, respectively. 
Specifically, the sample with a positive remanence value showed upward magnetic moments, staying at point $\mathrm{A}$ before all the measurements were performed. As shown in Fig. $2 c$ and $d$, in the temperature range of $50-300 \mathrm{~K}$, the magnetizations keep nearly flat, which results from the Fe sublattice. Thus, the positive sign indicates the upward direction of the total magnetic moments of the $\mathrm{Fe}$ sublattice. As the temperature decreases below $50 \mathrm{~K}$, the magnetization changes dramatically, likely resulting from the magnetic ordering of Gd moments [22,23]. Based on the effective field model, the $M(T)$ curves under different magnetic fields (Fig. $2 \mathrm{c}$ and d) below $50 \mathrm{~K}$ could be divided into three types. The effective field $B_{\text {eff }}$ applied to Gd atoms can be described as

$B_{\text {eff }}=B_{\mathrm{Fe}}+B_{\text {ex }}$,

where $B_{\mathrm{Fe}}$ and $B_{\mathrm{ex}}$ are the effective field from the $\mathrm{Fe}$ sublattice and the external magnetic field, respectively. The estimated value of $B_{\mathrm{Fe}}$ from $\mathrm{GdFeO}_{3}$ is about $\sim 310 \mathrm{Oe}$ $[6,24]$.

(1) When the absolute value of the $B_{\mathrm{ex}}$ is smaller than $100 \mathrm{Oe}$, the magnetization increases with the decrease of the temperature. In this case, the $B_{\mathrm{Fe}}$ that is relatively larger than $B_{\text {ex }}$ plays a more important role in the magnetic moments of the Gd sublattice. Thus, the magnetic moments of the Gd sublattice are induced in the same upward direction with the Fe sublattice, resulting in the sudden rise in the $M(T)$ curve at about $30 \mathrm{~K}$.

(2) When the value of $B_{\text {ex }}$ reaches $200 \mathrm{Oe}$, the magnetization exhibits the first decrease at $50 \mathrm{~K}$ to a minimum, and then a sharp increase at $T=5.2 \mathrm{~K}$. The $B_{\text {ex }}$ here becomes a major part of $B_{\text {eff }}$ and cannot be neglected. As the temperature decreases from $50 \mathrm{~K}$ to lower, the $\mathrm{Gd}$ atoms are partially affected by the external field with a downward direction and produce downward magnetic moments, leading to the lessening of the upward total magnetic moment and the decline of $M(T)$ curve. At $5.2 \mathrm{~K}$, more $\mathrm{Gd}$ atoms become magnetic-ordered and produce upward magnetic moments, in the same direction with the Fe sublattice and opposite to the applied field, leading to a sharp increase of magnetization.

(3) When the value of $B_{\mathrm{ex}}$ becomes $400 \mathrm{Oe}$, the external field is strong enough to overcome the $B_{\mathrm{Fe}}$, leading to the reverse to a large negative value (Fig. $2 \mathrm{~d}$ ). Thus, due to the strong effect of $B_{\mathrm{ex}}$ to the Gd sublattice at temperatures below $50 \mathrm{~K}$, the magnetic moment decreases first. Since the total magnetic anisotropy field is lower than the external field, it indicates that both moments of the $\mathrm{Fe}$ and Gd sublattices are magnetized along the applied field below $17 \mathrm{~K}$, where the total magnetic moments suddenly turn from a positive to a negative value. This is a distinctive spin-flip behavior which is not found in pure $\mathrm{GdFeO}_{3}[6,25]$.

The hysteresis loops under different temperatures were also measured with the measuring field applied along $b$ direction. As shown in Fig. 3a, each hysteresis loop contains a linear curve in a broad range of measuring fields and a small loop whose saturated magnetization is much smaller than the linear part. Notably, the magnetization of the loop switches sharply. This implies that the $\mathrm{Gd}_{0.75} \mathrm{La}_{0.25} \mathrm{FeO}_{3}$ is mainly AFM, but the canted ferromagnetic component survives due to the DM interaction. The extracted coercivity is plotted in Fig. 3b, which clearly shows that the coercivity is almost a constant above $50 \mathrm{~K}$ and declines when the temperature decreases below $50 \mathrm{~K}$. It is commonly accepted that the coercivity usually decreases with the increasing temperature, with very few exceptions. It is unique to have a type of material with a constant coercivity within a wide temperature range, whose stability is desired for the technical application. Based on the structure of $\mathrm{Gd}_{0.75} \mathrm{La}_{0.25} \mathrm{FeO}_{3}$, the Hamiltonian is as follows:
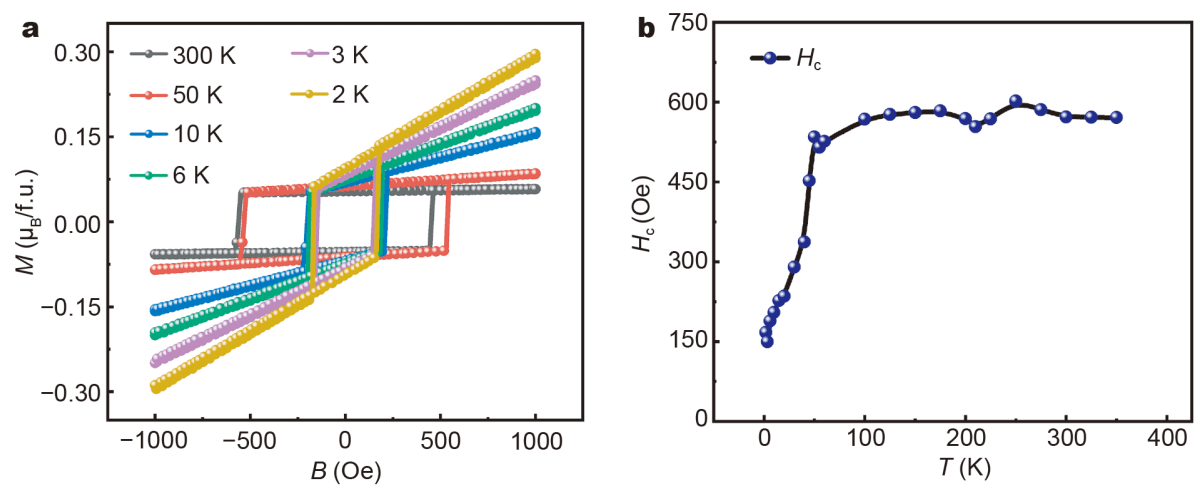

Figure 3 (a) Hysteresis Loops measured along b-direction. (b) Coercivity as a function of temperature. 


$$
\begin{aligned}
H= & \sum_{<i j>} J_{\mathrm{Fe}} S_{i} \cdot S_{j}+\sum_{<i j>} J_{\mathrm{Gd}} S_{i} \cdot S_{j} \\
& +\sum_{<i j>} J_{\mathrm{GdFe}} S_{i} \cdot S_{j}+\sum_{<i j>} D \cdot S_{i} \times S_{j} \\
& +\sum_{<i>} K_{2} S_{i z}^{2}+\sum_{<i>} B_{z} S_{i z},
\end{aligned}
$$

where the first and the second terms denote the exchange interaction of the Fe sublattice and Gd sublattice, respectively. The third term is the coupling between Fe and Gd sublattices. The DM interaction from the Gd sublattice has been added as the fourth one. The last two terms come from the ionic anisotropy and external magnetic field.

The Gd sublattice is paramagnetic in the high-temperature region. Thus, the high-temperature magnetic properties only stem from the Fe sublattice. By downfolding the mean effect of the $\mathrm{Gd}$ sublattice from the external field of Fe lattice, the Hamiltonian becomes

$$
\begin{aligned}
& H \approx \sum_{<i j>} \widetilde{J}_{\mathrm{Fe}} S_{i} \cdot S_{j}+\sum_{<i j>} D \cdot S_{i} \times S_{j} \\
& +\sum_{<i>} K_{2} S_{i z}^{2}+\sum_{<i>} \widetilde{B}_{z} S_{i z} .
\end{aligned}
$$

Some typical representative values of the parameters are selected without loss of generality. $\widetilde{\mathrm{Fe}}_{\mathrm{Fe}}, D, K_{2}$ and $\widetilde{B}_{z}$ are selected as 1 a.u., 0.05 a.u., $(0,0,0.05)$ a.u., and 0 respectively. The calculated total energy $v s$. spatially rotating angle of two Fe spins is plotted in Fig. 4a. The energy surface reveals a minimum when the spin directions of two Fe atoms are antiparallel. However, due to the DM interaction, the location of the energy minimum is not exactly at the position where two spin directions are antiparallel. Thus the ground state is not entirely AFM since the magnetic moments are canted along the $b$-axis, resulting in the $\mathrm{wFM}$ ordering. Considering the term of anisotropy, there is a barrier between two minima. A field large enough can overcome the barrier and switch wFM

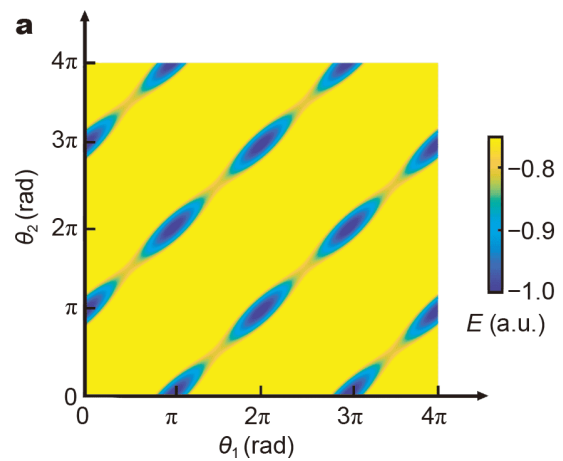

components, which leads to the distinctive spin-flip. Therefore, the magnetic states can be treated as two-fold quantum wells, where the magnetization is an analogy to a trapped quasiparticle. The barrier can be estimated by multiplying the saturated magnetic moment and magnetic field. The atomic doping can be used to adjust the barrier since the atomic doping could change the spinorbital coupling between $\mathrm{Fe}$ atoms. In Fig. $4 \mathrm{~b}$, the magnetic moments are trapped in either state of quantum wells. Hence, the coercivity and magnetic moment remain almost constant in the high-temperature region. In the low-temperature region with decreasing temperature, the magnetic ordering of $\mathrm{Gd}$ sublattices appears, yielding an effect on the total anisotropy field. Thus, the coercivity, the value of the external reverse field, reduces abruptly as the temperature decreases below $50 \mathrm{~K}$.

Based on the above phenomena, materials with similar states may be suitable for fabricating devices that work at room temperature, especially for non-volatile memory that retains information when the power is off. To estimate the potential application, the magnetic domains were measured by a magnetic force microscope (MFM) under different measuring fields at a temperature of $3.5 \mathrm{~K}$ (Fig. 5). There were clear changes in the magnetic domains when the measuring field was applied. For instance, the small measuring field of 100 Oe can affect the domains without a hitch. The written pattern can be also read without destroying when a small field was applied to the magnetic probe of MFM.

Notably, the induced magnetic moment of the Gd sublattice has the same direction with the Fe sublattice, different from the usual AFM coupling between the rareearth atom and $\mathrm{Fe}$ atom in $\mathrm{RFO}_{3}$ compounds $[1,6,10,26-$ 28]. In a similar material of $\mathrm{GdFeO}_{3}$, Das et al. [6] reported that the exchange interaction between the Gd and

b

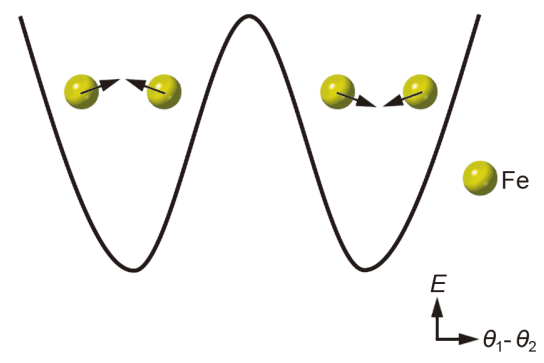

Figure 4 Illustration of the magnetic quantum wells in $\mathrm{Gd}_{0.75} \mathrm{La}_{0.25} \mathrm{FeO}_{3}$. (a) Calculated energy surface. $\theta_{1}$ and $\theta_{2}$ are the relative spatial angles of two Fe atoms from the $z$-axis in space. The color represents the energy $E$. (b) Illustration of the magnetic quantum wells and the corresponding magnetic configures of two Fe atoms. 

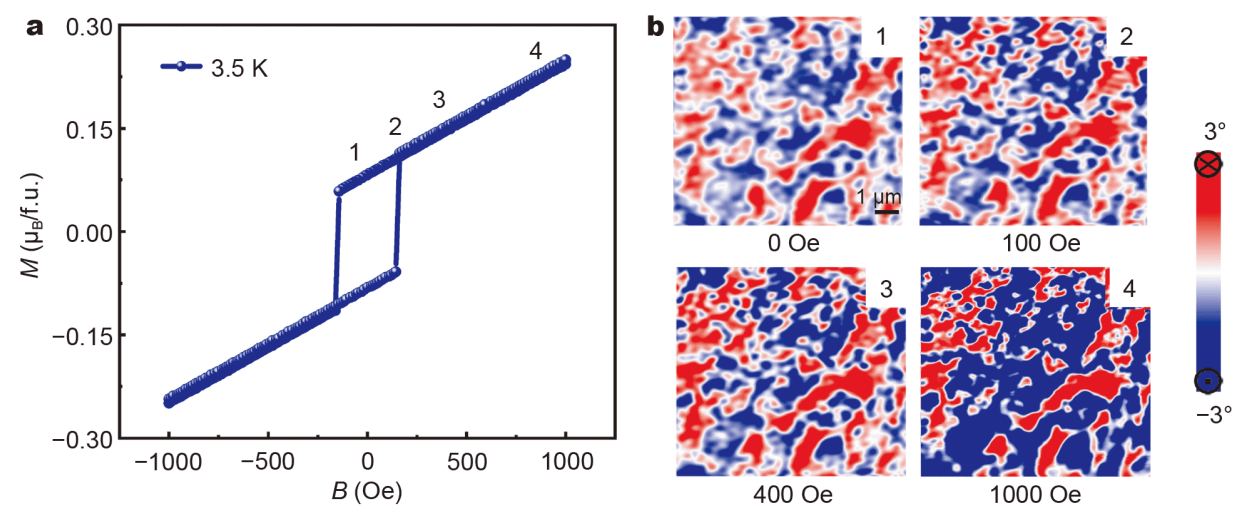

Figure 5 (a) Hysteresis loop at $3.5 \mathrm{~K}$. (b) Magnetic domains related to the hysteresis loop at $3.5 \mathrm{~K}$. The blue and red denote magnetic moments along up and down directions, respectively.

Fe moments produced an effective magnetic field at the $\mathrm{Gd}^{3+}$ site with the opposite direction to the canted $\mathrm{Fe}^{3+}$ moment. We also tested $\mathrm{GdFeO}_{3}$ with the same method as described above (see Figs S4 and S5), and the performance is the same as Das's report. As there are only $25 \%$ non-magnetic La atoms doping in the $\mathrm{GdFeO}_{3}$ and no structural phase transition, such a vast change in Gd-Fe coupling is unexpected. We thus propose that the paramagnetic La atoms replace the magnetic $\mathrm{Gd}$ atoms in a cell unit of $\mathrm{Gd}_{0.75} \mathrm{La}_{0.25} \mathrm{FeO}_{3}$, leading to the destruction of the coupling between the Gd and Fe sublattices. At low temperatures, $\sum_{<i j>} J_{\mathrm{Gd}} S_{i} \cdot S_{j}$ and $\sum_{<i j>} J_{\mathrm{GdFe}} S_{i} \cdot S_{j}$ are smaller than usual and depend on the way that La atoms replace the Gd sublattice. Therefore, the binary quantum well will still exist at low temperatures without the coupling between Gd and Fe sublattices. When there is no AFM coupling, the total magnetic moments of $\mathrm{Gd}_{0.75^{-}}$ $\mathrm{La}_{0.25} \mathrm{FeO}_{3}$ are easier to reverse than that of $\mathrm{GdFeO}_{3}$.

In summary, $\mathrm{Gd}_{0.75} \mathrm{La}_{0.25} \mathrm{FeO}_{3}$ is synthesized as a perfect single crystal with uniqueness in coercivity temperature coefficient, magnetization process, and spin-flip. The observed phenomena can be explained qualitatively by a Hamiltonian model based on the spin quantum well-like energy surface that can trap magnetic moments in a certain direction. $\mathrm{Gd}_{0.75} \mathrm{La}_{0.25} \mathrm{FeO}_{3}$ is easier to switch in the binary quantum well, being suitable for spintronic devices. Furthermore, the doping of La destroys the AFM coupling in Gd and Fe sublattices and promotes the influence of Fe sublattice-derived effective magnetic field on the Gd atoms.

Received 21 May 2020; accepted 24 June 2020; published online 28 August 2020

1 Tokunaga Y, Furukawa N, Sakai H, et al. Composite domain walls in a multiferroic perovskite ferrite. Nat Mater, 2009, 8: 558-562

2 Berini B, Mistrik J, Dumont Y, et al. Pulsed laser deposition and optical characterizations of the magnetic samarium orthoferrite. Thin Solid Films, 2012, 520: 1890-1894

3 Schmool DS, Keller N, Guyot M, et al. Magnetic and magnetooptic properties of orthoferrite thin films grown by pulsed-laser deposition. J Appl Phys, 1999, 86: 5712-5717

4 Kimel AV, Kirilyuk A, Tsvetkov A, et al. Laser-induced ultrafast spin reorientation in the antiferromagnet $\mathrm{TmFeO}_{3}$. Nature, 2004, 429: 850-853

5 Park BH, Kang BS, Bu SD, et al. Lanthanum-substituted bismuth titanate for use in non-volatile memories. Nature, 1999, 401: 682684

6 Das M, Roy S, Mandal P. Giant reversible magnetocaloric effect in a multiferroic $\mathrm{GdFeO}_{3}$ single crystal. Phys Rev B, 2017, 96: 174405

7 Lee WY, Yun HJ, Yoon JW. Characterization and magnetic properties of $\mathrm{LaFeO}_{3}$ nanofibers synthesized by electrospinning. J Alloys Compd, 2014, 583: 320-324

8 Shen H, Cheng Z, Hong F, et al. Magnetic field induced discontinuous spin reorientation in $\mathrm{ErFeO}_{3}$ single crystal. Appl Phys Lett, 2013, 103: 192404

9 Marshall LG, Cheng JG, Zhou JS, et al. Magnetic coupling between $\mathrm{Sm}^{3+}$ and the canted spin in an antiferromagnetic $\mathrm{SmFeO}_{3}$ single crystal. Phys Rev B, 2012, 86: 064417

10 Cao S, Zhao H, Kang B, et al. Temperature induced spin switching in $\mathrm{SmFeO}_{3}$ single crystal. Sci Rep, 2015, 4: 5960

11 Muralidharan R, Jang TH, Yang CH, et al. aMagnetic control of spin reorientation and magnetodielectric effect below the spin compensation temperature in $\mathrm{TmFeO}_{3}$. Appl Phys Lett, 2007, 90: 012506

12 Livingston JD. Present understanding of coercivity in cobalt-rare earths. AIP Conf Proc. 1973, 10: 643-657

13 Sagawa M, Hirosawa S, Tokuhara K, et al. Dependence of coercivity on the anisotropy field in the $\mathrm{Nd}_{2} \mathrm{Fe}_{14} \mathrm{~B}$-type sintered magnets. J Appl Phys, 1987, 61: 3559-3561

14 Kechrakos D, Trohidou KN. Magnetic properties of dipolar interacting single-domain particles. Phys Rev B, 1998, 58: 1216912177

15 Guo X, Chen X, Altounian Z, et al. Temperature dependence of coercivity in MnBi. J Appl Phys, 1993, 73: 6275-6277

16 Liu JF, Chui T, Dimitrov D, et al. Abnormal temperature depen- 
dence of intrinsic coercivity in $\mathrm{Sm}(\mathrm{Co}, \mathrm{Fe}, \mathrm{Cu}, \mathrm{Zr})_{z}$ powder materials. Appl Phys Lett, 1998, 73: 3007-3009

17 Rong C, Zhang H, Shen B, et al. Mechanism of the anomalous temperature dependence of coercivity in $\mathrm{Sm}(\mathrm{Co}, \mathrm{Fe}, \mathrm{Cu}, \mathrm{Zr})_{z}$ hightemperature magnets. Appl Phys Lett, 2006, 88: 042504

18 Zhou J, Skomski R, Chen C, et al. Sm-Co-Cu-Ti high-temperature permanent magnets. Appl Phys Lett, 2000, 77: 1514-1516

19 Geller S. Crystal structure of gadolinium orthoferrite, $\mathrm{GdFeO}_{3}$. J Chem Phys, 1956, 24: 1236-1239

20 Yuan SJ, Ren W, Hong F, et al. Spin switching and magnetization reversal in single-crystal $\mathrm{NdFeO}_{3}$. Phys Rev B, 2013, 87: 184405

21 Bousquet E, Cano A. Non-collinear magnetism in multiferroic perovskites. J Phys-Condens Matter, 2016, 28: 123001

22 Lee JH, Jeong YK, Park JH, et al. Spin-canting-induced improper ferroelectricity and spontaneous magnetization reversal in $\mathrm{SmFeO}_{3}$. Phys Rev Lett, 2011, 107: 117201

23 Zhao HJ, Íniguez J, Chen XM, et al. Origin of the magnetization and compensation temperature in rare-earth orthoferrites and orthochromates. Phys Rev B, 2016, 93: 014417

24 Cashion JD, Cooke AH, Hoel LA, et al. Magnetic properties of gadolinium ortho-vanadate. Proc R Soc London, 1970, 318: 473495

25 Paul P, Prajapat C L, Rajarajan A K, et al. Low temperature magnetic properties of $\mathrm{GdFeO}_{3}$. AIP Conference Proceedings, 2018, 1942. 130029

26 Scola J, Dumont $\mathrm{Y}$, Keller N, et al. Incomplete spin reorientation in yttrium orthoferrite. Phys Rev B, 2011, 84: 104429

27 Tokunaga M, Yamasaki Y, Onose Y, et al. Novel multiferroic state of $\mathrm{Eu}_{(1-x)} \mathrm{MnO}_{3}$ in high magnetic fields. Phys Rev Lett, 2009, 103: 187202

28 Nikolov O, Hall I, Barilo S N, et al. Field-induced spin reorientations in $\mathrm{TbFeO}_{3}$ at $4.2 \mathrm{~K}$. J Magn Magn Mater, 1996, 152: 75-85

Acknowledgements This work was supported by the National Key Research and Development Program of China (2016YFB0700901, 2017YFA0206303, 2017YFA0206301 and 2017YFA0403701), and the National Natural Science Foundation of China $(51731001,11675006$ and 11805006). The authors thank Dr. Y. Zhang (State Key Laboratory of Artificial Microstructures and Mesoscopic Sciences) for the magnetometry and Research Fellow L. Zhang (Steady High Magnetic Field Facilities, High Magnetic Field Laboratory, CAS) and Research Associate W. H. Zhu (Institute of Physics, CAS) for the measurement in the XRD. This paper utilized the resources of the Center for Electron Microscopy at Peking University.

Author contributions Wen X and Yang J conceived the idea. Fang Y, Cao $\mathrm{S}$, Zhang $\mathrm{X}$, Cheng $\mathrm{Z}$ prepared the materials. Song $\mathrm{Z}$ conducted the DFT calculations. Malik IA and Zhang J conducted the measurement of magnetic domains. Wen $\mathrm{X}$ performed the experiments and wrote the paper with support from Yang J. Yang W, Han J, Liu S, Du H and Hou Y contributed to the correction of the manuscript. Hou Y and Yang J revised the manuscript. All authors contributed to the general discussion.

Conflict of interest The authors declare that they have no conflict of interest.

Supplementary information Experimental details and supporting data are available in the online version of the paper.

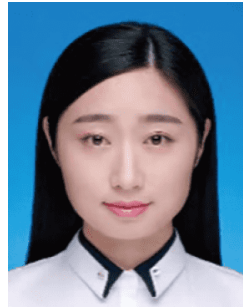

Xin Wen is currently a $\mathrm{PhD}$ candidate at the School of Physics, Peking University (PKU). Her research interests include the magnetic materials such as rare earth orthoferrites.

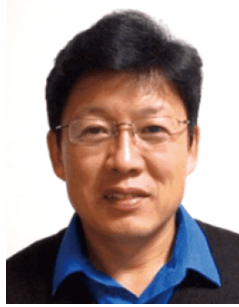

Jinbo Yang is a professor and a leader of the Center for Magnetism and Magnetics in the School of Physics in PKU. He received his PhD in condensed matter physics from PKU in 1998 and performed post-doctoral research at IFWDresden Germany from 1998 to 2000. He then worked at the University of Missouri-Rolla (UMR) from 2001 to 2008. He became a full professor at PKU in 2008. His research interests focus on the structure and magnetism in condensed matter, including hard/soft magnets, crystal, magnetic and electronic structures of magnetic materials, nanomagnetism and spintronics.

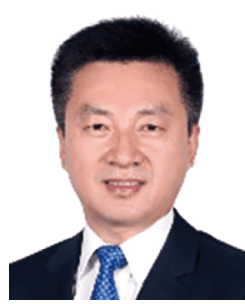

Yanglong Hou obtained his $\mathrm{PhD}$ degree in materials science from Harbin Institute of Technology (China) in 2000. After a short postdoctoral training at $\mathrm{PKU}$, he worked at the University of Tokyo from 2002 to 2005 as a JSPS foreign special researcher and also at Brown University from 2005 to 2007 as a postdoctoral researcher. He joined PKU in 2007, and now is a Chang Jiang Chair Professor of Materials Science. His research interests include the design and chemical synthesis of functional nanoparticles, and their biomedical and energy-related applications.

\section{单晶 $\mathrm{Gd}_{0.75} \mathrm{La}_{0.25} \mathrm{FeO}_{3}$ 中的类自旋量子阱行为}

闻馨 ${ }^{1}$, 宋志刚 ${ }^{1}$, Iftikhar Ahmed Malik ${ }^{2}$, 方依霏 ${ }^{3}$, 杨文云 ${ }^{1,4}$, 韩景智 ${ }^{1,4}$, 刘顺荃 ${ }^{1,4}$, 杜红林 ${ }^{1,4}$, 曹世勋 ${ }^{5}$, 张金星 ${ }^{2}$, 张向群 ${ }^{6}$, 成昭华 ${ }^{6}$, 侯仰龙 ${ }^{4,7^{*}}$, 杨金波 $^{1,5^{*}}$

摘要 为满足实际应用, 自旋电子学器件需要自旋在特定方向发生 自发取向, 但这通常是难以控制的. 在本文中, 我们观察了沿 $b$ 轴的 单晶 $\mathrm{Gd}_{0.75} \mathrm{La}_{0.25} \mathrm{FeO}_{3}$ 在室温下伴随着弱铁磁 $(\mathrm{wFM}$ ) 成分的反铁磁 (AFM)磁有序. 与普通的AFM材料不同, 这里的 $w F M$ 磁矩是离散的, 类似于二维量子阴, 可以通过一个小磁场在两个状态之间切换. 量 子阱中的势垒使得矫顽力在 50-350 K的宽温度范围内保持稳定, 并 在 $17 \mathrm{~K}$ 时产生独特的自旋翻转现象. 此外, 在 $\mathrm{RFeO}_{3}(\mathrm{R}=$ 稀土)中, 稀 土和铁的次晶格之间普遍存在着一种AFM耦合. 而在 $\mathrm{Gd}_{0.75} \mathrm{La}_{0.25}$ $\mathrm{FeO}_{3}$ 中, $\mathrm{Gd}$ 与 Fe次晶格之间的 $\mathrm{AFM}$ 耦合被破坏，使得 $\mathrm{Gd}$ 被诱导出 的磁矩与 $\mathrm{Fe}$ 磁矩方向不再相反, 而是表现出与 $\mathrm{Fe}$ 磁矩方向相同的诱 导磁矩. 由于在 $\mathrm{GdFeO}_{3}$ 或 $\mathrm{LaFeO}_{3}$ 中不存在自旋翻转, 因此AFM耦 合的破坏也被认为是材料表现出一种独特的自旋翻转现象的主要 原因. 以上性质使得 $\mathrm{Gd}_{0.75} \mathrm{La}_{0.25} \mathrm{FeO}_{3}$ 在信息存储方面具有更优良的 可控性. 